\title{
Malignant Lymphoma, Large Cell, Cleaved
}

National Cancer Institute

\section{Source}

National Cancer Institute. Malignant Lymphoma, Large Cell, Cleaved. NCI Thesaurus. Code C27265.

Antiquated term describing non-Hodgkin lymphomas that are usually diffuse and are composed of large cells with irregular nuclei, invisible nucleoli and a small amount of cytoplasm. This morphologic category includes both mature B- and mature T-cell lymphomas.--2004 